ARTICLE

https://doi.org/10.1038/s41467-019-10371-1

\title{
Vibrio sp. dhg as a platform for the biorefinery of brown macroalgae
}

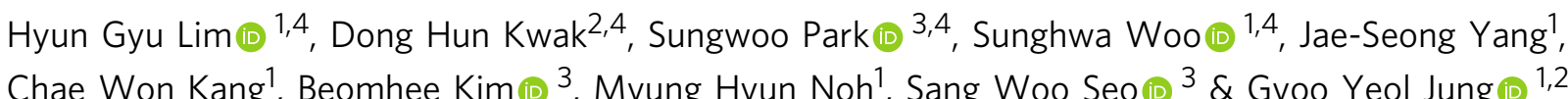

Although brown macroalgae holds potential as an alternative feedstock, its utilization by conventional microbial platforms has been limited due to the inability to metabolize one of the principal sugars, alginate. Here, we isolate Vibrio sp. dhg, a fast-growing bacterium that can efficiently assimilate alginate. Based on systematic characterization of the genomic information of Vibrio sp. dhg, we establish a genetic toolbox for its engineering. We also demonstrate its ability to rapidly produce ethanol, 2,3-butanediol, and lycopene from brown macroalgae sugar mixture with high productivities and yields. Collectively, Vibrio sp. dhg can be used as a platform for the efficient conversion of brown macroalgae sugars into diverse value-added biochemicals.

\footnotetext{
${ }^{1}$ Department of Chemical Engineering, Pohang University of Science and Technology, 77 Cheongam-Ro, Nam-Gu, Pohang, Gyeongbuk 37673 , Korea. ${ }^{2}$ School of Interdisciplinary Bioscience and Bioengineering, Pohang University of Science and Technology, 77 Cheongam-Ro, Nam-Gu, Pohang, Gyeongbuk 37673, Korea. ${ }^{3}$ School of Chemical and Biological Engineering, Institute of Chemical Process, Seoul National University, 1 Gwanak-Ro, Gwanak-Gu, Seoul 08826, Korea. ${ }^{4}$ These authors contributed equally: Hyun Gyu Lim, Dong Hun Kwak, Sungwoo Park, Sunghwa Woo. Correspondence and requests for materials should be addressed to S.W.S. (email: swseo@snu.ac.kr) or to G.Y.J. (email: gyjung@postech.ac.kr)
} 
T he global demand for bioproducts is increasing at a striking rate, with its market share expected to reach $22 \%$ of the chemical industry by $2025^{1}$. To meet such a vast demand, stable supplementation and conversion of feedstocks have been critical over the past years. Although starch crops have been widely used up until now, many concerns exist regarding the consumption of food resources and limited cultivation capabilities. In this respect, brown macroalgae have been suggested as an alternative feedstock. Brown macroalgae are hugely abundant and have a high carbohydrate content (35-60\% of dry weight $)^{2-4}$. They can grow much faster than lignocellulosic biomasses and only require sunlight and seawater ${ }^{5}$.

The most prominent sugars in brown macroalgae are alginate (a copolymer of $\alpha$-L-guluronate and $\beta$-D-mannuronate) and mannitol. While a conventional microbial platform (e.g., Escherichia coli) can easily metabolize mannitol, its ability to assimilate alginate is hindered by the fact that it lacks certain related genes; it is known that alginate metabolism requires about 10-20 genes that encode transporters, lyases, and metabolic enzymes ${ }^{6,7}$. Although recent studies have demonstrated that $E$. coli can be engineered to utilize alginate with introduction a huge gene cluster for alginate utilization from naturally-occurring alginate-metabolizing microorganism ${ }^{6,8}$, their growth rates and metabolic activities are still too low for industrial applications, likely due to the unoptimized expression of multiple xenogeneic genes.

As both the growth rate and the metabolic activity of host microorganisms greatly affect the performance of bioprocesses, it is crucial to exploit an efficient host with high rates of both these factors in order to obtain a high productivity. Therefore, microorganisms that are naturally capable of using alginate should be considered as the preferred microbial platform for brown macroalgae feedstocks. Due to natural optimization throughout evolutionary history, such microorganisms likely have a superior capacity to metabolize alginate compared with engineered versions of conventional microbes. Thus, these naturally occurring microorganisms would be more suitable as a microbial platform for producing diverse value-added biochemicals from the sugars of brown macroalgae.

In this study, we isolate a fast-growing microorganism capable of utilizing alginate efficiently. We name this microorganism as Vibrio sp. dhg and characterize it systematically to develop a genetic engineering toolbox. By exploitation as a microbial platform, we demonstrate diverse value-added biochemical production from brown macroalgae sugars with high productivities and yields. From these results, we propose Vibrio sp. dhg as a platform for the biorefinery of brown macroalgae.

\section{Results}

Isolation of an alginate-utilizing microorganism. To isolate alginate-utilizing microorganism, seaweed sludge was collected and inoculated in minimal medium supplemented with alginate as a sole carbon source (Supplementary Note 1). After a few rounds of sub-culturing at $30^{\circ} \mathrm{C}$, a rod-shaped microorganism showing a rapid growth (maximum specific growth rate $(\mu)=$ $0.98 \mathrm{~h}^{-1}$ ) was successfully isolated (Fig. 1a). This microorganism is able to use not only alginate but also other biomass-derivable sugars (e.g., glucose, mannitol, sucrose, galactose, arabinose, and glycerol). Notably, with most sugars, the rates of growth and sugar uptake at $30^{\circ} \mathrm{C}$ were substantially higher or at least comparable with those of $E$. coli grown with glucose at $37^{\circ} \mathrm{C}$ (Fig. 1b, c).

In addition, this microorganism can grow even in a high concentration of salt ( $100 \mathrm{~g} \mathrm{~L}^{-1}$ of sodium chloride) while other industrial hosts (E. coli, Corynebacterium glutamicum,
Saccharomyces cerevisiae, Supplementary Data 1, and Supplementary Fig. 1a) showed severely reduced growth rates. Tolerance to other representative bioproducts (ethanol, 2,3-butanediol (2,3$\mathrm{BDO}$ ), and lactate) was also investigated (Supplementary Fig. 1b-d). In the case of ethanol, this microorganism was similar to C. glutamicum and maintained its growth with $50 \mathrm{~g} \mathrm{~L}^{-1}$ of ethanol in the medium. For 2,3-BDO, it was similar to E. coli but more sensitive than C. glutamicum and S. cerevisiae; the growth was inhibited by $50 \mathrm{~g} \mathrm{~L}^{-1}$ of 2,3-BDO. Finally, with lactate, its tolerance was slightly less than the tolerance of S. cerevisiae which showed the best growth in the presence of $50 \mathrm{~g}$ $\mathrm{L}^{-1}$ of lactate. Overall, although its tolerance was not much superior to either C. glutamicum or S. cerevisiae, it was comparable to E. coli, suggesting this microorganism can be used as a host for biochemical production.

To identify this microorganism, we analyzed its $16 \mathrm{~S}$ rDNA sequence using the universal primers (Supplementary Data 2) and found that it belongs to the family Vibrionaceae. In particular, it was highly similar to Vibrio natriegens ATCC14048 (99\% identity, Supplementary Data 3), a gram-negative bacterium recently suggested to be a promising host for molecular biotechnology due to its rapid growth rate (doubling time $<10$ $\mathrm{min}$ in rich medium $)^{9,10}$. Upon sequencing the genome of our isolated microorganism (Supplementary Fig. 2, Supplementary Tables 1 and 2), three circular contigs representing a large chromosome, a small chromosome, and a plasmid were obtained. Although several Vibrio species are known to use alginate (e.g., $V$. alginolyticus and $V$. splendidus 12B01), its growth rate is much higher than reported values of the known microorganisms $\left(0.2-0.8 \mathrm{~h}^{-1}\right)^{7}$. Moreover, the genome context and phenotypic characteristics of our microorganism were closer to those of $V$. natriegens ATCC14048 (Supplementary Figs. 3 and 4). However, detailed physiological comparison (Supplementary Table 3) revealed that there is a difference between two microorganisms, probably due to a variation in more than $5 \%$ of their genomes. Particularly, V. natriegens does not possess the machinery needed to metabolize alginate. Therefore, we named our strain Vibrio sp. dhg and further investigated its potential as a platform for biorefinery from brown macroalgae.

Genome annotation revealed that many of the genes responsible for alginate assimilation in Vibrio sp. dhg were located in a 42-kb cluster within its small chromosome (chromosome 2, Supplementary Data 4). Sequence comparison with a well-studied gene cluster of $V$. splendidus ${ }^{6}$ indicated that our strain also assimilates alginate via a multi-step process (Fig. 1a). Specifically, endolytic alginate lyases are secreted to depolymerize alginate into oligo-alginates ${ }^{11}$. The oligo-alginates are then transported into the cytosol through a transporter. Subsequently, alginate monomers (4-deoxy-L-erythro-5-hexoseulose uronate, DEHU) are produced by the action of exolytic oligoalginate lyases. Finally, via a partial Entner-Doudoroff pathway, DEHU reductase (DehR), 2-keto-3-deoxygluconate kinase (KdgK) and 2-keto-3-deoxyphosphogluconate aldolase (Eda) convert DEHU into glyceraldehyde-3-phosphate and pyruvate, two metabolites that then enter glycolysis. On the other hand, the large chromosome contains two copies of the mannitol operon (chromosome 1). Mannitol is initially transported into the cell by a mannitol-specific phosphotransferase system (PTS, MtlA) and then converted into fructose-6-phosphate-an intermediate of glycolysis-by mannitol-1-phosphate dehydrogenase (MtlD).

To confirm that the aforementioned genes are critical for alginate and mannitol assimilation, we analyzed their expression levels via RNA sequencing. As expected, these genes were highly induced by the addition of the cognate sugar (up to a 100 -fold increase compared with the glucose condition) 
a

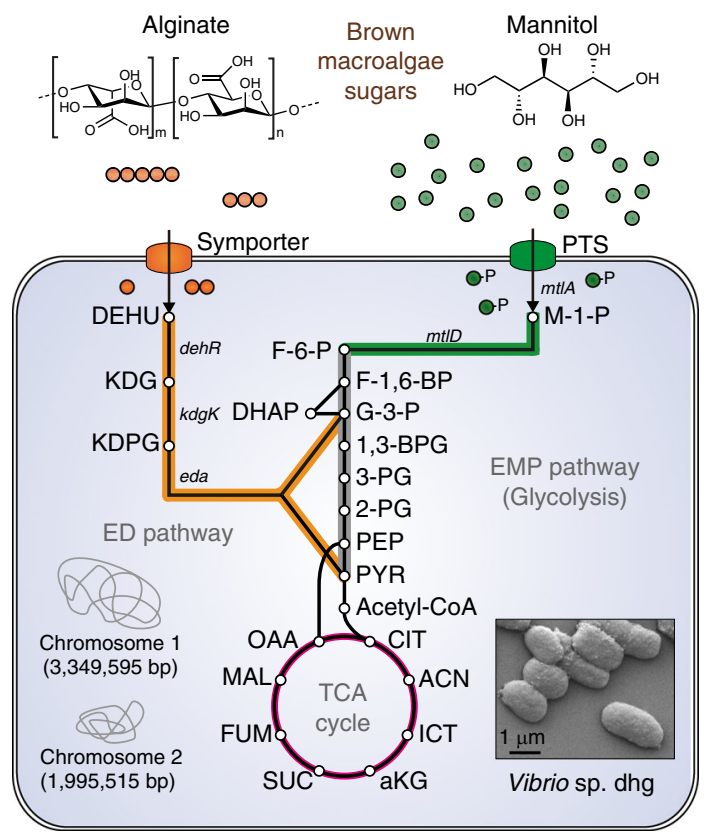

b

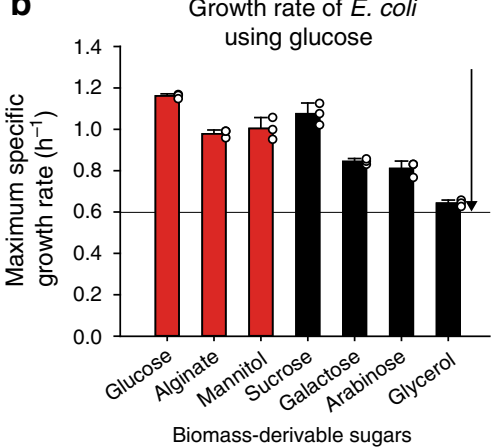

d

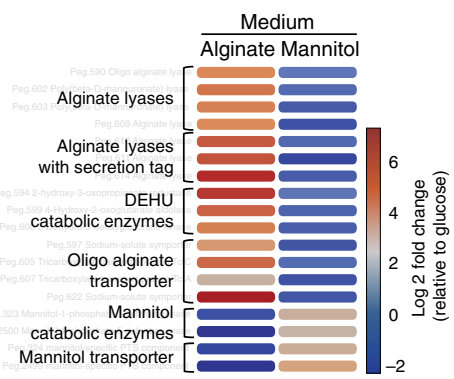

C

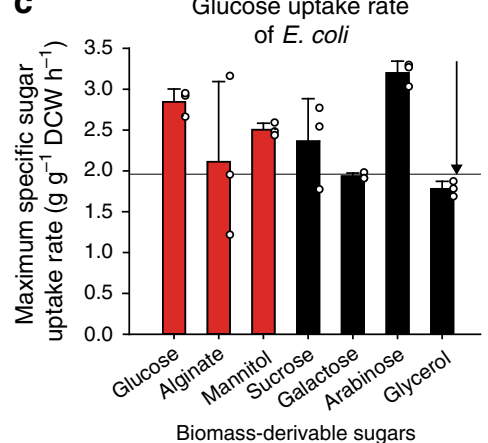

e

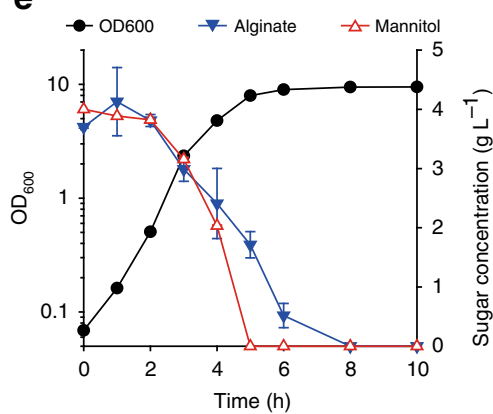

Fig. 1 Vibrio sp. dhg as a microbial platform for brown macroalgae biorefinery. a Schematic alginate and mannitol assimilation pathways in Vibrio sp. dhg. Depolymerized oligo-alginate is transported into the cytosol and digested into 4-deoxy-L-erythro-5-hexoseulose uronate (DEHU). DEHU is further converted to glyceraldehyde 3-phosphate (G-3-P) and pyruvate (PYR). Mannitol is transported into the cytosol by the PTS system and is converted to fructose-6-phosphate (F-6-P). The inset is a scanning electron microscopy (SEM) image of Vibrio sp. dhg with a scale bar indicating $1 \mu \mathrm{m}$. The other abbreviations are as follows: ED pathway Entner-Doudoroff pathway, EMP pathway Embden-Meyerhof-Parnas pathway, KDG 2-keto-3-deoxygluconate, KDPG 2-keto-3-deoxy-6-phosphogluconate, M-1-P mannitol-1-phosphate, F-1,6-BP fructose 1,6-bisphosphatase, DHAP dihydroxyacetone phosphate, 1,3BPG 1,3-bisphosphoglycerate, 3-PG 3-phosphoglycerate, 2-PG 2-phosphoglycerate, PEP phosphoenolpyruvate, CIT citrate ACN cis-aconitate, ICT isocitrate, aKG a-ketoglutarate, SUC succinate, FUM fumarate, MAL malate, and OAA oxaloacetate. b Maximum specific growth rates ( $\mathrm{h}^{-1}$ ) and $\mathbf{c}$ maximum sugar uptake rates $\left(\mathrm{g} \mathrm{g}^{-1}\right.$ dry cell weight $(\mathrm{DCW}) \mathrm{h}^{-1}$ ) of Vibrio sp. dhg in minimal medium supplemented with $4 \mathrm{~g} \mathrm{~L}^{-1}$ of diverse carbon sources at $30^{\circ} \mathrm{C}$. The two horizontal lines indicate growth rate $\left(0.60 \mathrm{~h}^{-1}\right)$ and sugar uptake rate $\left(1.96 \mathrm{~g} \mathrm{~g}^{-1} \mathrm{DCW} \mathrm{h}{ }^{-1}\right)$ of $E$. coli in glucose minimal medium at $37^{\circ} \mathrm{C}$. d Fold changes of gene expression during alginate and mannitol assimilation as compared with during glucose assimilation, based on RNA-Seq results.

e Simultaneous assimilation of alginate and mannitol by Vibrio sp. dhg. Closed black circles, $\mathrm{OD}_{600}$; closed inverted triangles, alginate; open red triangles, mannitol. Error bar indicates the standard deviations of three independent cultures $(n=3)$. White dot indicates actual data point. Source data of Figs. 1b, $1 c$, and $1 \mathrm{~d}$ are provided as a Source Data file

(Fig. 1d). Additionally, we observed that Vibrio sp. dhg could simultaneously consume alginate and mannitol (Fig. 1e). As these two sugars have different oxidation states, this property is highly advantageous for biochemical production (e.g., ethanol) in an oxygen-limited environment (see Supplementary Note 2) ${ }^{12,13}$. Thus, Vibrio sp. dhg is highly suited to using an alginatemannitol mixture.

Gene expression control in Vibrio sp. dhg. To harness Vibrio sp. dhg as a microbial platform, genetic engineering tools for the construction and optimization of (heterologous) metabolic pathways must first be readily available. As an elemental approach, we introduced several plasmids with different origins of replication (pMB1, RSF1030, p15A, and CloDF13) into Vibrio sp. dhg strain. Using a transformation protocol originally established for $V$. natriegens ${ }^{10}$, we observed that all plasmids except CloDF13 could stably exist (Supplementary Fig. 5). Furthermore, more than two different plasmids could be maintained under the presence of multiple antibiotics.

Next, we investigated the features of transcription that drive gene expression in Vibrio sp. dhg. Upon comparing the amino acid sequence of its housekeeping sigma factor $\left(\sigma_{70}\right)$ with that of E. coli, we noticed that the major DNA-binding domains (region 2.4 and 4.2) were 100\% identical (Supplementary Fig. 6).
Furthermore, the promoter regions of ribosomal genes, which are highly expressed in Vibrio sp. dhg, also have similar sequences (TTGANN and TATAAT) to the bacterial -35 and -10 boxes (TTGACA and TATAAT, respectively) (Supplementary Fig. 7). These findings suggested that the common promoters used for $E$. coli would also be compatible with Vibrio sp. dhg. To test this idea, we prepared plasmids encoding the $s g f p$ (super-folding green fluorescence protein) gene under the control of the conventional promoters $\mathrm{P}_{\text {J23119 }}, \mathrm{P}_{\text {lac }}, \mathrm{P}_{\text {tac }}, \mathrm{P}_{\text {tet }}$, and $\mathrm{P}_{\text {ara }}$. These plasmids also carry cognate repressor expression cassettes (lacI and araC cassettes from $E$. coli, and a synthetically designed tet $R$ cassette) for inducible expression. As expected, we were able to observe successful expression of the $s g f p$ gene in Vibrio sp. dhg when transcribed by the endogenous RNA polymerase (Fig. 2a). In addition, expression of the T7 RNA polymerase gene (under the control of the $\mathrm{P}_{\text {lac }}$ promoter) also resulted in the expression of $s g f p$ when placed under the control of the $\mathrm{P}_{\mathrm{T} 7}$ promoter. Furthermore, all inducible promoters showed strong activation (up to a maximum of 184-fold) upon the addition of cognate inducers (isopropyl $\beta$-D-1-thiogalactopyranoside (IPTG), anhydrotetracycline (aTc) or arabinose).

To achieve precise control of gene expression at the transcriptional level, we constructed a synthetic promoter library. This library was prepared by modifying the -35 and -10 boxes of the $\mathrm{P}_{\mathrm{J} 23119}$ promoter with degenerate sequences (i.e., YTKAYR 
a
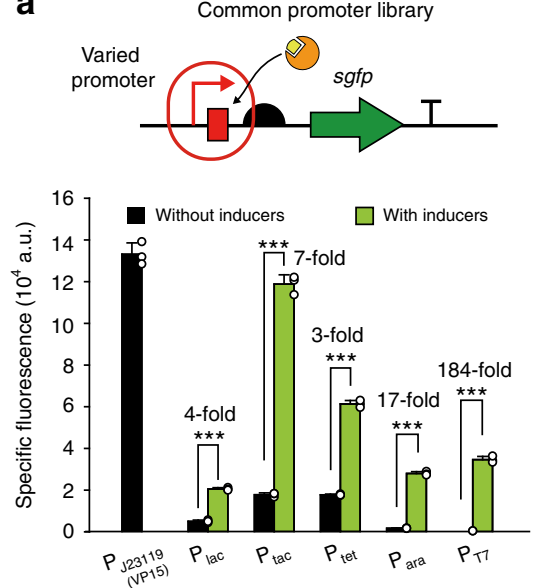

b
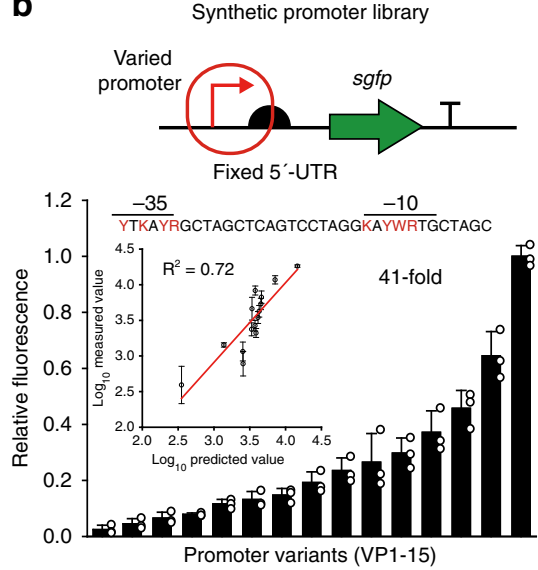

c

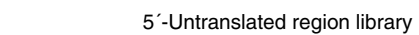

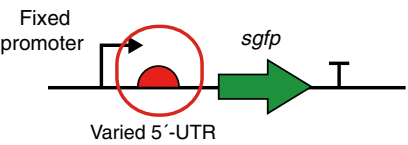

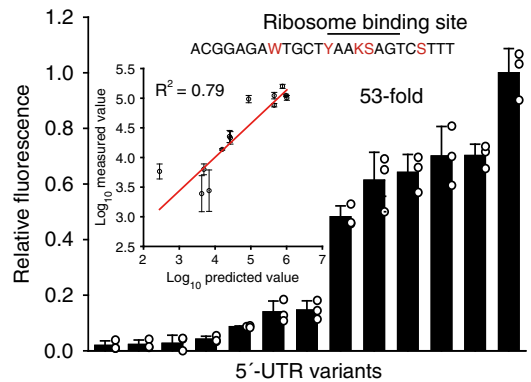

Fig. 2 Precise control of gene expression in Vibrio sp. dhg. a Evaluation of a constitutive promoter $\left(P_{J 23119}\right.$, equivalent to $\left.P_{V P 15}\right)$ and various inducible promoters $\left(P_{\text {lac }}, P_{\text {tac }}, P_{\text {tet }}, P_{\text {ara, }}\right.$ and $\left.P_{\text {T7 }}\right)$ in terms of sgfp expression. For inducible expression, either $1 \mathrm{mM} \mathrm{IPTG,} 100 \mu \mathrm{g} \mathrm{mL}^{-1}$ aTc, or $10 \mathrm{~g} \mathrm{~L}^{-1}$ arabinose was added. ${ }^{\star \star \star} p<0.001$ (two-sided $t$-test). b Relative fluorescence values for sgfp expression under the control of various synthetic promoters. Each fluorescence value was normalized by the highest value. The inset graph indicates a positive correlation $\left(R^{2}=0.72\right)$ between the predicted promoter strength and the measured fluorescence values. c Relative fluorescence values for sgfp expression in function of various $5^{\prime}$-UTRs. Each fluorescence value was normalized by the highest value. The inset graph indicates a high correlation $\left(R^{2}=0.79\right)$ between the predicted folding energies $\left(\Delta \mathrm{G}_{U T R}\right)$ and the measured fluorescence values. Values were adjusted for background fluorescence (fluorescence of the VDHG001 strain). Error bar indicates the standard deviations of three independent cultures $(n=3)$. White dot indicates actual data point. Source data of Figs. 2a, 2b, and 2c are provided as a Source Data file

and KAYWRT, respectively) to cover the Anderson promoter library ${ }^{14}$. By altering the promoter sequence, we successfully obtained 15 different promoters (named promoters VP1 to 15) of varied strengths, with a maximum fold difference of 41 (Fig. 2b, Supplementary Table 4). Based on our data, we were able to construct a predictive model that can estimate promoter strengths (Supplementary Table 5) with a strong predictive power $\left(R^{2}=\right.$ 0.72 ). In agreement with the observed strengths, our model predicted promoter VP15 to be the strongest. Thus, our model could be useful in the development of other synthetic promoters and be further improved by iterative-learning.

We also investigated gene expression control in Vibrio sp. dhg at the translational level. Recently, prediction of translation efficiency in bacteria became possible thanks to a biophysical model of the translation initiation process ${ }^{15,16}$. This model, which has been thoroughly tested in E. coli, calculates the thermodynamic free energy change of the $5^{\prime}$-untranslated region $\left(\Delta \mathrm{G}_{\mathrm{UTR}}\right)$. Although the $16 \mathrm{~S}$ rDNA of Vibrio sp. dhg (Supplementary Data 3 ) has a high similarity $(90 \%)$ and the same antiShine-Dalgarno sequence (ACCTCCTTA) to the $16 \mathrm{~S}$ rDNA of $E$. coli, the biophysical model still requires validation in Vibrio sp. dhg. Thus, to confirm the predictable nature of translational efficiency in Vibrio sp. dhg, we generated a $5^{\prime}$-UTR library covering a broad range of sgfp expression levels using UTR Library Designer ${ }^{16}$ (Supplementary Table 6). In total, we analyzed 13 5'-UTR variants and found that gene expression was successfully varied. More specifically, alteration of the ribosome binding site and the folding features ${ }^{16}$ enabled us to generate variants with a difference in expression of up to 53-fold (Fig. 2c). More importantly, these measured values were highly correlated $\left(R^{2}=0.79\right)$ with the predicted values, thereby indicating a strong predictive power for this model in Vibrio sp. dhg. Taken together, both transcription and translation can be controlled in a predictable manner in Vibrio sp. dhg, a property that is highly useful for the construction of efficient metabolic pathways. Furthermore, many plasmid-based production systems or genetic circuits, which have been previously developed for E. coli, can be directly employed in Vibrio sp. dhg without any major modifications.
Genome editing in Vibrio sp. dhg. As genome editing is necessary to develop an efficient cell factory, the possibility to engineer the genome of Vibrio sp. dhg was explored. To engineer its genome, the SXT recombination system naturally found in Vibrionaceae was chosen. It has been reported that the expression of exo (a gene encoding alkaline exonuclease) and beta (a gene encoding single-strand annealing protein) permits recombination in $V$. natriegens and $E$. coli ${ }^{17,18}$. For its genome engineering, endogenous exo and beta in Vibrio sp. dhg, were expressed under the control of the $\mathrm{P}_{\text {tac }}$ promoter and a synthetic 5'-UTR (Supplementary Table 7). Additionally, gamma (a gene encoding nuclease inhibitor) from lambda phage was expressed to facilitate recombination ${ }^{17}$. As a template for allelic exchange, we introduced a chloramphenicol resistance (cat) cassette flanked with two identical FRT sequences ${ }^{19}$ and homology arms (1-3 kb) of the target locus ${ }^{10}$. After electroporation, we confirmed the engineered locus by checking for colonies that could grow on a chloramphenicol agar plate. Indeed, we found colonies with target gene successfully replaced by the cat gene (Supplementary Fig. 8a). Subsequently, the integrated cat gene was easily removed by expressing an FRT-specific flippase from S. cerevisiae ${ }^{19}$.

Biochemical production from alginate and mannitol mixture. After establishing a genetic toolbox for Vibrio sp. dhg, we attempted to produce value-added biochemicals using engineered Vibrio sp. strains. Although sugar composition of brown macroalgae is known to be influenced by geographical and seasonal changes ${ }^{20}$, a mixture of alginate and mannitol in a $1: 2$ ratio was chosen to prepare a mimetic sugar medium to fully evaluate its performance. At first, production of ethanol, one of biofuels, was attempted in a microaerobic condition (Fig. 3a). Since the wildtype strain possesses both an aldehyde dehydrogenase and an alcohol dehydrogenase, ethanol can be produced by cultivating the wildtype strain via reduction of acetylCoA. However, only $1.6 \mathrm{~g} \mathrm{~L}^{-1}$ of ethanol was produced from 30 $\mathrm{g} \mathrm{L}^{-1}$ of mixed sugars (Supplementary Fig. 9). In addition, 
a
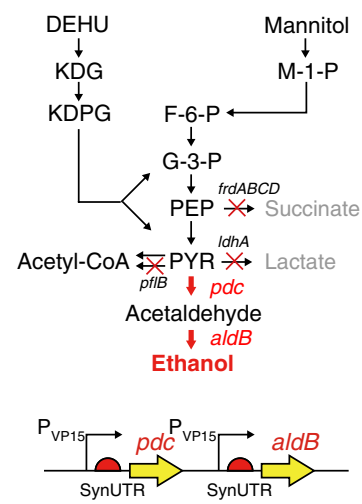

b

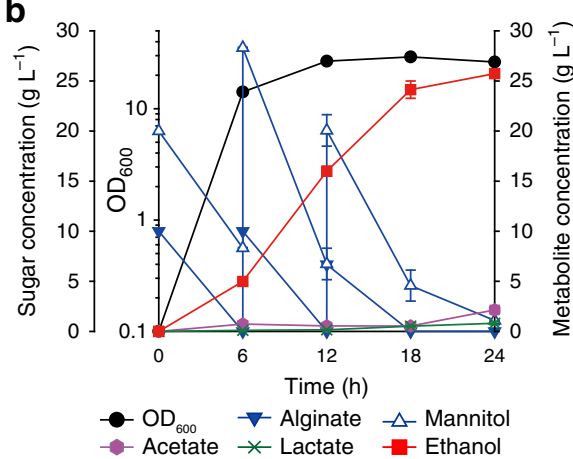

C

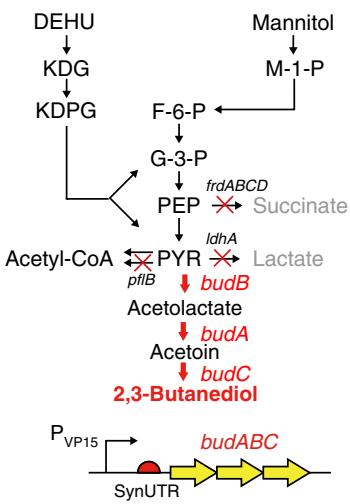

e

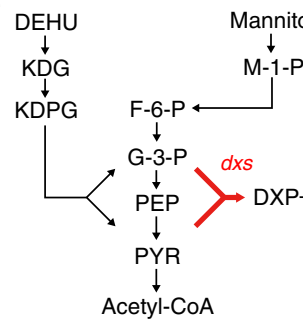

HMBPP

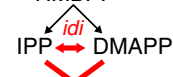

Acetyl-CoA

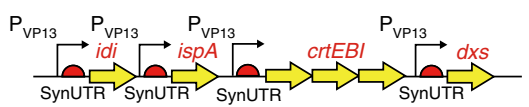

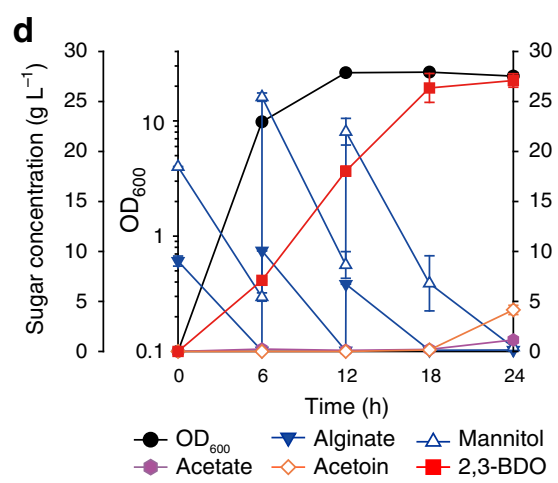

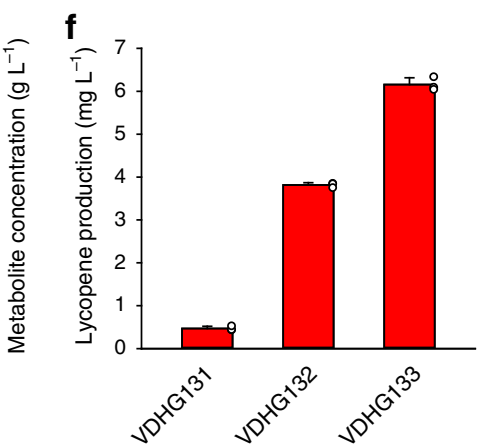

Fig. 3 Biochemical production using engineered Vibrio sp. dhg strains. a Ethanol production pathway. For ethanol production, pdc (pyruvate decarboxylase) and aldB (aldehyde dehydrogenase) from Z. mobilis were expressed under the PVP15 promoter and a synthetic 5'-UTR. To improve yield, IdhA, frdABCD, and pfIB were removed from the chromosome. $\mathbf{b}$ Fermentation profile of the VDHG 411 strain during $24 \mathrm{~h}$. Closed black circle, $\mathrm{OD}_{600}$; closed inverted blue triangle, alginate; open blue triangle, mannitol; closed purple hexagon, acetate; green cross, lactate; closed red square, ethanol. c 2,3-BDO production pathway. The budABC operon from E. aerogenes was expressed under the $\mathrm{P}_{\text {VP15 }}$ promoter and a synthetic $5^{\prime}$-UTR. d Fermentation profile of the VDHG421 strain during $24 \mathrm{~h}$. Closed black circle, $\mathrm{OD}_{600}$; closed inverted blue triangle, alginate; open blue triangle, mannitol; closed purple hexagon, acetate; open orange diamond, acetoin; closed red square, 2,3-BDO. e Lycopene production pathway. For lycopene production, the crtEBl operon (encoding GGPP synthase, phytoene synthase, and phytoene desaturase) from L. purpurea was expressed under the PVP13 promoter and a synthetic 5'-UTR. To improve the titer, $d x s$ (DXS synthase), idi (IPP isomerase) and ispA (FPP syntase) from E. coli W3110 were additionally expressed. $\mathbf{f}$ Lycopene production by engineered Vibrio sp. dhg strains (VDHG131, VDHG132, and VDHG133) after $9 \mathrm{~h}$. Abbreviations: DXP deoxyxylulose 5-phosphate, HMBPP 4-hydroxy-3methylbut-2-enyl pyrophosphate, IPP isopentenyl pyrophosphate, DMAPP dimethylallyl pyrophosphate, FPP farnesyl diphosphate, GGPP geranylgeranyl pyrophosphate. Some reactions of glycolysis have been omitted for simplicity. Error bar indicates the standard deviations of three independent cultures $(n=3)$. White dot indicates actual data point

other by-products such as acetate, lactate, and succinate were produced in considerable amounts (a total of $4.7 \mathrm{~g} \mathrm{~L}^{-1}$ ). To produce more ethanol, the pyruvate decarboxylase (pdc) and aldehyde dehydrogenase (aldB) genes of Zymomonas mobils ${ }^{6}$ (Fig. 3a) were expressed. To obtain constitutive expression at the maximum possible level, we fused each gene to a synthetic $5^{\prime}$-UTR (Supplementary Table 7) and placed them under the control of the strongest synthetic promoter, VP15. The resulting strain showed dramatically improved ethanol production $\left(7.7 \mathrm{~g} \mathrm{~L}^{-1}\right.$, or a 4.8 -fold increase) and lower levels of accumulated by-products (a total of $2.3 \mathrm{~g} \mathrm{~L}^{-1}$ ). To further minimize by-product formation, we deleted the endogenous $l d h A$ (lactate dehydrogenase), $f r d A B C D$ (fumarate reductase) and $p f l B$ (pyruvate-formate lyase) genes, creating the VDHG411 strain (Supplementary Fig. 8b). This deletion successfully minimized the loss of carbon (a total of only $1.1 \mathrm{~g} \mathrm{~L}^{-1}$ of by-products) while slightly improving ethanol production (8.4 $\mathrm{g} \mathrm{L}^{-1}$, or a $10 \%$ increase). Upon providing a total of $80 \mathrm{~g} \mathrm{~L}^{-1}$ of mixed sugars with a 1:2 ratio by fed-batch fermentation, VDHG411 strain produced $25.7 \mathrm{~g} \mathrm{~L}^{-1}(3.3 \% \mathrm{v} / \mathrm{v})$ of ethanol in $24 \mathrm{~h}$ (Fig. 3b). Surprisingly, this $1.1 \mathrm{~g} \mathrm{~L}^{-1} \mathrm{~h}^{-1}$ average ethanol productivity (maximum $1.8 \mathrm{~g} \mathrm{~L}^{-1} \mathrm{~h}^{-1}$ ) was dramatically higher than that of other microbial platforms for alginate utilization
(Supplementary Table 8 ). Furthermore, the yield (64\% of the theoretical maximum) of our VDHG411 strain was comparable to the yield of other alginate-consuming microbial platforms.

We next attempted to produce 2,3-BDO, a chemical that can be used industrially as an antifreeze reagent or solvent ${ }^{21}$ (Fig. 3c). Although the production of 2,3-BDO from the sugars of brown macroalgae has previously been attempted ${ }^{21}$, this was carried out using E. coli, which is only able to slowly assimilate mannitol. In contrast, alginate has not yet been considered as a carbon source in such a bioprocess. Thus, to produce 2,3-BDO using our alginate-assimilating Vibrio sp. dhg, we expressed the budABC operon (encoding for acetolactate decarboxylase, acetolactate synthase, and acetoin reductase) from Enterobacter aerogenes ${ }^{21}$ under the $\mathrm{P}_{\mathrm{VP} 15}$ promoter and a synthetic 5'-UTR (Supplementary Table 7). The heterologous expression of this operon enabled the production of $6.1 \mathrm{~g} \mathrm{~L}^{-1}$ of 2,3-BDO from $30 \mathrm{~g} \mathrm{~L}^{-1}$ of mixed sugars in $9 \mathrm{~h}$ (Supplementary Fig. 10). Moreover, by deleting the competing pathways (i.e., $\triangle l d h A, \triangle f r d A B C D$, and $\triangle p f l B$ ), we were able to further improve production 1.5 -fold $\left(9.4 \mathrm{~g} \mathrm{~L}^{-1}\right.$, the VDHG421 strain). By fed-batch fermentation, this strain was able to produce $27.1 \mathrm{~g} \mathrm{~L}^{-1}$ of $2,3-\mathrm{BDO}\left(31.3 \mathrm{~g} \mathrm{~L}^{-1}\right.$ including acetoin) from $80 \mathrm{~g} \mathrm{~L}^{-1}$ of sugars in $24 \mathrm{~h}$ (Fig. 3d). When 
compared with the $E$. coli platform of the previously mentioned study $^{21}$, Vibrio sp. dhg showed both superior productivity $(1.3 \mathrm{~g}$ of 2,3-BDO and acetoin $\left.\mathrm{L}^{-1} \mathrm{~h}^{-1}\right)$ and yield ( $0.40 \mathrm{~g}$ product $\mathrm{g}^{-1}$ sugar or $81 \%$ of the theoretical maximum), along with an efficient co-utilization of alginate and mannitol.

We lastly demonstrated the ability of our Vibrio sp. dhg platform to produce lycopene, which is a C-40 phytochemical used as a nutraceutical antioxidant ${ }^{22,23}$. We first prepared the required plasmids (Supplementary Data 1) by re-organizing previously reported plasmids ${ }^{23}$ that express crtEBI (geranylgeranyl pyrophosphate (GGPP) synthase, phytoene synthase and phytoene desaturase) from Lamprocystis purpurea, and $d x s$ (1deoxy-D-xylulose-5-phosphate (DXP) synthase), idi (isopentenyl diphosphate (IPP) isomerase) and ispA (farnesyl diphosphate (FPP) synthase) from E. coli (Fig. 3e). These genes were expressed under the control of the $\mathrm{P}_{\mathrm{VP} 13}$ promoter and synthetic $5^{\prime}$-UTRs ${ }^{23}$ (Supplementary Table 7). When we supplemented strain VDHG131-the strain expressing only the essential lycopene genes (crtEBI) - with $10 \mathrm{~g} \mathrm{~L}^{-1}$ of sugar mixture, $0.47 \mathrm{mg} \mathrm{L}^{-1}$ of lycopene was produced in $9 \mathrm{~h}$ (Fig. $3 \mathrm{f}$ ). Additional expression of idi and ispA (strain VDHG132) or idi, ispA, and $d x s$ (strain VDHG133) successfully increased lycopene production ( $3.8 \mathrm{mg} \mathrm{L}^{-1}$ and $6.2 \mathrm{mg} \mathrm{L}^{-1}$ for the two strains, respectively). Compared with a similarly constructed $E$. coli strain $\left(\sim 4 \mathrm{mg} \mathrm{L}^{-1}\right.$ during $24 \mathrm{~h})^{23}$, the use of Vibrio sp. dhg enabled a much faster production of lycopene (more than a fourfold increase in productivity). As slow growth and low accumulation of biomasses are known hurdles for efficient production of lycopene, the fastgrowing Vibrio sp. dhg could be an ideal host. Importantly, additional engineering - such as precursor balancing ${ }^{23}$ - using our developed toolbox could enhance lycopene production even further.

Brown macroalgae fermentation for ethanol production. Finally, we tested ethanol production using brown macroalgae as feedstock (Fig. 4). The sugar content of used Kelp powder was quantified to $0.16 \mathrm{~g}$ alginate and $0.3 \mathrm{~g}$ mannitol per $\mathrm{g}$ powder (see

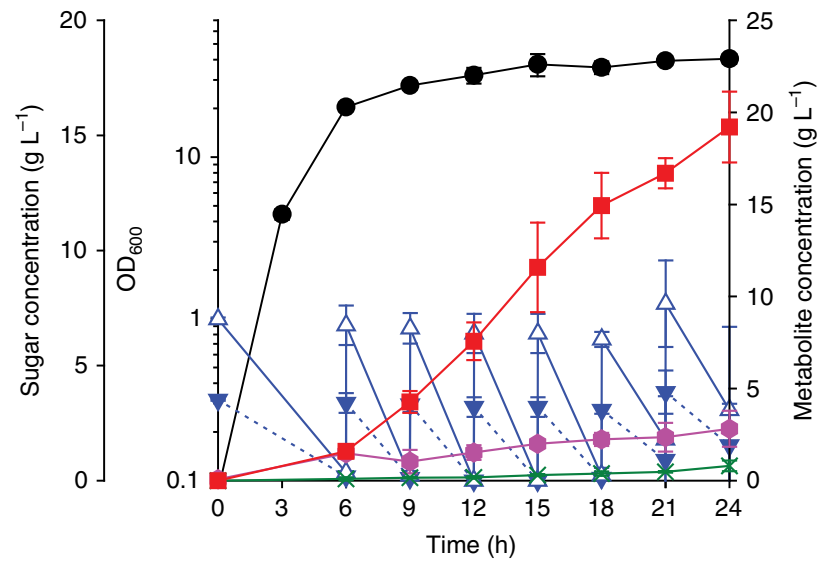

$-\mathrm{OD}_{600}-\boldsymbol{\nabla}$ - Alginate $\triangle$ Mannitol - Acetate $*$ Lactate $\rightarrow$ Ethanol

Fig. 4 Brown macroalgae fermentation for ethanol production. A brown macroalgae fermentation profile of the VDHG411 strain using a $5 \mathrm{~L}$ bioreactor during $24 \mathrm{~h}$. Residual alginate concentration was roughly estimated based on mannitol concentration using the measured kelp composition due to difficulty in its quantification using concentrated kelp medium. $\mathrm{OD}_{600}$ values after $6 \mathrm{~h}$ may not represent the exact amount of biomass due to turbidity from the Kelp powder. Closed black circle, $\mathrm{OD}_{600}$; closed inverted blue triangle, alginate; open blue triangle, mannitol; closed purple hexagon, acetate; green cross, lactate; closed red square, ethanol. Error bar indicates the standard deviations of three independent cultures $(n=3)$
"Methods"), consistent with the composition of the mimetic sugar mixture. Therefore, the Kelp powder can be directly used as a feedstock. When the VDHG411 strain was cultivated in a bioreactor containing a $1 \mathrm{~L}$ medium, total $120 \mathrm{~g} \mathrm{~L}^{-1}$ of the Kelp powder was provided during $24 \mathrm{~h}$. Subsequently, even without any enzymatic pre-treatment or hydrolysis, $19.2 \mathrm{~g} \mathrm{~L}^{-1}$ of ethanol $(2.4 \% \mathrm{v} / \mathrm{v})$ was successfully produced with a similar yield $(63 \%$ of theoretical maximum) compared to the yield from mimetic sugar fermentation. Although the productivity $\left(0.8 \mathrm{~g} \mathrm{~L}^{-1} \mathrm{~h}^{-1}\right)$ was reduced, suggesting a requirement of further optimization including genetic engineering and parameter study, the obtained result clearly indicates that Vibrio sp. dhg is also capable to produce a biochemical directly from brown macroalgae.

\section{Discussion}

The ultimate goal of the biorefinery process is the efficient conversion of abundant carbon sources into value-added biochemicals. While the use of brown macroalgae as a feedstock has been limited due to the difficulties of alginate assimilation, the use of Vibrio sp. dhg strain as a microbial platform could overcome this issue. Moreover, its outstanding growth rate and metabolic activity over conventional platforms allow envisioning accelerated biochemical production from brown macroalgae.

One risk in exploiting environmental isolates as a host is their potential virulence. Our genome analysis revealed that Vibrio sp. dhg strain does not have major toxins found from pathogenic Vibrio strains ${ }^{10}$ (see "Methods"). However, one barely expressed open reading frame (fragments per kilobase per million (FPKM) $<1$ based on RNA-seq analysis) showed a marginal identity $(24 \%$, Supplementary Fig. 11a and b) when compared with its amino acid sequence with an auxiliary toxin (Zot, zona occludens toxin). Although it has a low sequence identity (less than $30 \%{ }^{24}$ ) and Vibrio sp. dhg strain does not have two major toxins (CtxA and CtxB) concurrently found with $\mathrm{Zot}^{25}$, we tested that the potential toxin can be deleted to minimize the risk. We confirmed that this gene can be deleted and does not affect the growth of the strain (Supplementary Fig. 11c and d).

For the realization of the brown macroalgae biorefinery, there could still be many hurdles. As mentioned, one challenge is a potential fluctuation of bioprocess efficiency due to seasonal and geographical differences in biomass sugar composition. Indeed, we observed that the ratio of alginate and mannitol, which have different oxidation states, hugely affected ethanol production (up to 4.5 -fold) (Supplementary Fig. 12 and Supplementary Note 2). To address this issue, microorganisms should be engineered for dynamic response to intracellular redox state for robust biochemical production with advantages of recent advances in synthetic biology 26-29.

Further studies aimed at unveiling the characteristics (e.g., rapid growth, halophilicity) and improving its tolerance ${ }^{30,31}$ of Vibrio sp. dhg would be valuable for maximizing its potential as a microbial platform ${ }^{32}$. Additionally, the exploitation of fast-growing microorganisms as hosts can also expedite evolutionary processes that lead to the development of improved proteins or microorganisms themselves. The strain development repertoire that we demonstrate here, could also be applied to the construction of other microbial platforms that utilize non-traditional carbon sources ${ }^{33,34}$. By combining all these efforts, we will likely be able to achieve the ultimate goal of the biorefinery in the near future.

\section{Methods}

Chemical reagents and oligonucleotides. Primers were synthesized by Cosmogenetech (Seoul, Korea) and are listed in Supplementary Data 2. Plasmid and genomic DNA were prepared using the GeneAll ${ }^{\mathrm{R}}$ Exprep $^{\mathrm{TM}}$ Plasmid SV kit and the Exgene ${ }^{\mathrm{TM}}$ Cell SV kit (GeneAll, Seoul, Korea), respectively. Procedures for plasmid cloning are explained in Supplementary Note 3. For purification of 
fragmented DNA, we used the Expin ${ }^{\mathrm{TM}}$ Gel SV and Expin ${ }^{\mathrm{TM}}$ PCR SV kits. Q5 polymerase, the NEBuilder ${ }^{\mathrm{R}} \mathrm{HiFi}$ DNA Assembly Cloning Kit, restriction enzymes, and the Quick Ligation ${ }^{\mathrm{TM}}$ kit were purchased from New England Biolabs (Ipswich, MA, United States). For routine colony PCR, EmeraldAmp ${ }^{\oplus}$ GT PCR Master Mix was used (Clontech, Mountain View, CA, USA). All reagents for cell cultures were purchased from BD Bioscience (Sparks, MD, USA). Chemical reagents were purchased from Sigma (St. Louis, MO, USA) unless otherwise stated. Buffer and medium compositions were described in Supplementary Note 1.

Isolation of Vibrio sp. dhg strain. To isolate an alginate-metabolizing microorganism, we obtained seaweed sludge from the coastal area of Pohang, Korea. The sludge sample was inoculated into $20 \mathrm{~mL}$ of alginate minimal medium in a $350-\mathrm{mL}$ flask. When growth was detected by a change in turbidity, the culture was transferred to the same fresh medium using a $1 / 100$ dilution. After several sub-culture, the broth was streaked on alginate agar plate. We sequenced the $16 \mathrm{~S}$ rDNA of 10 colonies using the 16S_27F and 16S_1492R primers (Supplementary Data 2). All samples had an identical sequence and one of the sequenced colonies was named Vibrio sp. dhg.

Strain characterization. For routine cell cultures, colonies were picked from rich medium agar plates (LBv2 medium for Vibrio sp. dhg, LB medium for E. coli, BHI medium for C. glutamicum, and YPD medium for S. cerevisiae) and inoculated in 3 $\mathrm{mL}$ of minimal medium (buffered medium for Vibrio sp. dhg, M9 medium for $E$. coli, CG medium for C. glutamicum, and SC medium for S. cerevisiae) supplemented with $4 \mathrm{~g} \mathrm{~L}^{-1}$ of a single sugar (glucose, mannitol, alginate, sucrose, arabinose, galactose, or glycerol). After growing overnight, the culture was refreshed by inoculating into fresh medium at an $\mathrm{OD}_{600}$ of $0.05-0.1$. When the $\mathrm{OD}_{600}$ reached 1.0, the culture broth was transferred into either 350-mL flask (aerobic culture) containing $20 \mathrm{~mL}$ of medium or $175-\mathrm{mL}$ serum bottle (anaerobic culture) containing $50 \mathrm{~mL}$ of medium. The cultures were conducted in a rotary shaker (Hanil Scientific, Gimpo, Korea) at $30^{\circ} \mathrm{C}$ and $200 \mathrm{rpm}$ (rotation per minute) unless otherwise mentioned. Especially, cells were handled in the anaerobic chamber (Coy Laboratories, Ann Arbor, MI, USA) when cells were anaerobically cultivated.

For the characterization of Vibrio sp. dhg in a reactor-scale, the refreshed seed was inoculated into a $5 \mathrm{~L}$ bioreactor (LiFlus GX, Hanil Scientific) containing the $1 \mathrm{~L}$ buffered medium with $10 \mathrm{~g} \mathrm{~L}^{-1}$ of glucose. $2 \mathrm{~L} \mathrm{~min}^{-1}$ of sterile air was provided and culture was stirred at $500 \mathrm{rpm}$. For an anaerobic culture, $50 \mathrm{mM}$ of $\mathrm{NaHCO}_{3}$ was additionally supplemented and $\mathrm{CO}_{2}$ and $\mathrm{N}_{2}$ gas mixture (20:80) was provided instead of air.

To test tolerance of the microorganisms, cells were cultured in $100-\mu \mathrm{L}$ minimal media contained in 96-well microtiter plate with orbital shaking (300 rpm). Different amounts $\left(0,10,20,50\right.$, and $\left.100 \mathrm{~g} \mathrm{~L}^{-1}\right)$ of sodium chloride, ethanol, lactate, 2,3-BDO were included in the medium. Lactate was pre-neutralized with sodium hydroxide. The growth of cells was measured using the Hidex Sense microplate reader (Hidex, Turku, Finland).

All cell cultures were conducted in biological triplicates. Initial $\mathrm{OD}_{600}$ for all cell culture was $0.05-0.1$. An $\mathrm{OD}_{600}$ of 1.0 corresponds to $0.27 \mathrm{~g}$ DCW per $\mathrm{L}^{9}$. Maximum specific growth rate $\left(\mu, h^{-1}\right)$ was calculated by linear regression of $\ln$ $\left(\mathrm{OD}_{600}\right)$ and time $(\mathrm{h})$ during exponential growth phase. To determine maximum specific sugar uptake rate $\left(\mathrm{g} \mathrm{g}^{-1} \mathrm{DCW} \mathrm{L}{ }^{-1}\right)$, maximum specific growth rate was divided by biomass yield ${ }^{9}$.

Scanning electron microscopy. Cells were cultured overnight in LBv2 medium and then sub-cultured in fresh medium by diluting 100-fold. Upon reaching an $\mathrm{OD}_{600}$ of 0.6 , the cells were collected by centrifugation at $3700 \times g$ for $10 \mathrm{~min}$ and washed three times with buffer solution $(100 \mathrm{mM}$ of potassium phosphate and $10 \mathrm{~g} \mathrm{~L}^{-1}$ of $\mathrm{NaCl}$ ). Subsequently, cells were fixed by incubation in the same solution containing $2.5 \%$ glutaraldehyde. After $1 \mathrm{~h}$ incubation, the cell pellet was harvested by centrifugation. The resulting pellet was resuspended in increasing concentrations of ethanol $(30,50,70,80$, and $100 \% \mathrm{v} / \mathrm{v})$ in the same buffer for dehydration. Then, $1 \mathrm{~mL}$ of suspension was dropped on a slide glass and dried at room temperature to remove ethanol. The sample was then coated with Pt in the presence of argon gas using a metal sputter. Finally, the sample was imaged at a $5 \mathrm{kV}$ acceleration voltage on a Jeol scanning electron microscope (JEOL JSM-7401F).

Annotation of the Vibrio sp. dhg genome sequence. To construct a draft of the Vibrio sp. dhg genome, purified genomic DNA (gDNA) was treated with the SMRT cell 8Pac v3 and DNA Polymerase Binding Kit P6 (Pacific Biosciences, CA, USA) and analyzed using the PacBio sequencer at Macrogen (Seoul, Korea). We obtained a total of 147,285 reads for 1,302,603,996 bases and then de novo assembled the reads using RS HGAP Assembly (v3.0) with default options. To correct for errors in the draft genome generated by the PacBio sequencer, we additionally used the Illumina short-read sequencer. A gDNA library was prepared using the KAPA HyperPlus Kit according to the manufacturer's instructions (KAPA Biosystems, Wilmington, MA, USA). This short gDNA library was sequenced using the MiniSeq 300-cycle mid-output kit (Illumina, San Diego, CA, USA). Paired-end reads obtained from MiniSeq were mapped onto the assembled genome from PacBio data using the breseq pipeline ${ }^{35}$ with default options. Predicted mutations were further validated by Sanger sequencing. All errors were listed in Table S5. The final versions of each contig were uploaded to GenBank (accession numbers: CP028943,
CP028944, and CP028945). The re-sequenced genome was annotated using Rapid Annotations of Subsystem Technology ${ }^{36}$.

RNA-Seq. For expression profiling, Vibrio sp. dhg was cultured in buffered minimal medium supplemented with alginate, mannitol, or glucose as a sole carbon source, and harvested at early exponential phase $\left(\mathrm{OD}_{600}=0.6\right)$. Total RNA including small RNAs was extracted by treating the cells with RNAprotect Bacteria Reagent (Qiagen, Hilden, Germany) and using an RNeasy Plus Mini kit (Qiagen, Hilden, Germany) according to the manufacturer's instructions. These samples were prepared in biological duplicates. The concentrations of total RNA were measured using a Thermo NanoDrop One spectrophotometer (Waltham, MA, USA) and the quality of the samples was assessed using a Bioanalyzer with the RNA 6000 Pico kit from Agilent Technologies (Santa Clara, CA, USA). Then, the ribosomal RNAs were removed from the total RNA using the Ribo-Zero rRNA Removal Kit for Gram-negative bacteria (Illumina, San Diego, USA). The quality of the rRNA-free samples was also checked using the Bioanalyzer with the RNA 6000 Pico kit. The strand-specific RNA-seq library was constructed using the KAPA Stranded RNA-Seq Library Preparation Kit for Illumina platforms according to the manufacturer's instructions. The size distribution of the complementary DNA library was assessed using the Bioanalyzer with a High Sensitivity DNA kit from Agilent Technologies. The samples were sequenced using the MiniSeq 75-cycle High-output Kit (Illumina, San Diego, USA) according to the manufacturer's instructions. The quality of the sequence reads obtained from RNA-seq was checked by FastQC ${ }^{37}$, and then aligned to the modified reference genome using bowtie $2^{38}$. The Cufflink package (http://cufflinks.cbcb.umd.edu/) $)^{39}$ was run to obtain transcriptomic data.

Consensus promoter sequence annotation. To identify consensus promoter sequences of endogenous genes, we piled up the mRNA fragments from RNA-Seq reads. The mapped reads were extended to the fragment length and accumulated. For mapping, we used bowtie 2 with default parameters ${ }^{38}$. Regions containing transcription start sites were extracted from the pile-up distribution for each gene We manually inspected the upstream sequences of ribosomal genes and identified the promoter sequences. All the inspected promoter sequences were then aligned by CLUSTALW and a sequence logo was generated using Web logo (https:// weblogo.berkeley.edu/logo.cgi).

Genetic engineering of Vibrio sp. dhg. Purified plasmids were transformed into Vibrio sp. dhg strain via a previously established electroporation method ${ }^{10}$. Briefly, a single colony was inoculated into $3 \mathrm{~mL}$ of LBv2 medium. When the medium became turbid, the culture was then transferred into $10 \mathrm{~mL}$ of fresh medium using a $1 / 100$ dilution. When an $\mathrm{OD}_{600}$ of 0.6 was reached, the culture was incubated in an icebox for $10 \mathrm{~min}$. Subsequently, a cell pellet was prepared by centrifugation at $13,000 \times g$ for $1 \mathrm{~min}$ at $4{ }^{\circ} \mathrm{C}$. This pellet was washed twice with electroporation buffer and resuspended to achieve an $\mathrm{OD}_{600}$ of 16 . Then, more than $100 \mathrm{ng}$ of plasmid DNA was added to $90 \mu \mathrm{L}$ of cell suspension and $0.8 \mathrm{kV}$ was applied for 2.5 ms using a micropulse-electroporator (Bio-Rad Laboratories, Richmond, CA, USA). The cells were recovered by addition of $1 \mathrm{~mL}$ of $\mathrm{BHI}$ recovery medium and incubated at $37^{\circ} \mathrm{C}$. Finally, the cells were spread on antibiotic-containing LBv2 agar plates. Plasmid introduction was confirmed by agarose gel or colony PCR using primers that specifically anneal to the plasmid DNA.

For genome engineering, we first made electro-competent cells using the cells expressing SXT recombinases (following the same protocol used for plasmid transformation). During sub-culture, $1 \mathrm{mM}$ of IPTG was included. For allelic exchange, more than $10 \mu \mathrm{g}$ of the dsDNA fragment was added to the cell suspension. The cells were recovered by incubating in BHI recovery medium for 3 $\mathrm{h}$ at $37^{\circ} \mathrm{C}$. Then, cells were spread on an agar plate containing $10 \mathrm{mg} \mathrm{L}^{-1}$ of chloramphenicol. The integrated selection marker was removed via the activity of FLP flippase ${ }^{19}$. The change of target locus was confirmed by colony PCR and Sanger sequencing.

Fluorescence measurements. To measure the fluorescence of each strain, colonies were inoculated into $180 \mu \mathrm{L}$ of buffered minimal medium containing $4 \mathrm{~g} \mathrm{~L}^{-1}$ of glucose using BioscreenC (Growth Curves, Helsinki, Finland). After two rounds of subculture, the fluorescences of $100-\mu \mathrm{L}$ samples were analyzed using the VICTOR ${ }^{3}$ 1420 Multilabel Plate Reader (Perkin Elmer, MA, Waltham, USA). Specific fluorescence was calculated by dividing the fluorescence by the $\mathrm{OD}_{600}$. For inducible promoters, different concentrations of inducers were included at time zero (1 of $\mathrm{mM}$ IPTG, $100 \mu \mathrm{g} \mathrm{L}^{-1}$ of aTc, or $10 \mathrm{~g} \mathrm{~L}^{-1}$ of arabinose).

Synthetic promoter strength prediction. To construct a predictive model from the synthetic promoters, we first converted the promoter sequences into a set of binary vectors. Each nucleotide was set as $(1,0,0,0),(0,1,0,0),(0,0,1,0)$, or $(0,0$, $0,1)$ for ' $A$ ', ' $C$ ', ' $G$ ', or ' $T$ ', respectively. Then, we used the Ridge regression model to determine the coefficients and predict promoter strengths. The Ridge regression model addresses the ordinary least squares problem to determine the coefficients that minimize a residual sum of squares and the size of coefficients as shown below:

$$
\min \left(\|X w-y\|_{2}^{2}+\alpha\|w\|_{2}^{2}\right)
$$


where $X$ denotes the vector-transformed promoter sequences, $y$ denotes the promoter strengths, and $w$ is a coefficient vector. $\alpha$ is a complexity parameter that balances the weight of emphasis given to minimizing the residual sum of squares and minimizing the sum of the square of coefficients. We performed leave-one-out cross-validation (LOOCV) in order to estimate how accurately the predictive model performs in practice. For each synthetic promoter sequence, we made a predictive model with the other sequences as a training set (i.e., the target sequence was excluded). For the implementation, we used the linear_model module from the sklearn package of python.

Biochemical production. Biochemical production (ethanol, 2,3-BDO, and lycopene) from mimetic sugar mixture was conducted using the buffered minimal medium, however, the concentration of $\mathrm{NaCl}$ was reduced to $10 \mathrm{~g} \mathrm{~L}^{-1}$. In the case of 2,3-BDO, an additional $5 \mathrm{~g} \mathrm{~L}^{-1}$ of yeast extract was included in the medium. The medium was supplemented with $30 \mathrm{~g} \mathrm{~L}^{-1}$ of an alginate-mannitol mixture (1:2 ratio) as carbon source. When $80 \mathrm{~g} \mathrm{~L}^{-1}$ of sugar was provided, $30 \mathrm{~g} \mathrm{~L}^{-1}$ and $20 \mathrm{~g} \mathrm{~L}^{-1}$ of the sugar mixture were additionally added at $6 \mathrm{~h}$ and $12 \mathrm{~h}$. Cells were cultivated in either $50 \mathrm{~mL}$ (for ethanol production) or $25 \mathrm{~mL}$ (for 2,3-BDO and lycopene production) of medium in a $350-\mathrm{mL}$ flask at $200 \mathrm{rpm}$.

For ethanol production from brown macroalgae, a powder of kelp (60 mesh) harvested in Korea was purchased from a local market. Its sugar composition was quantified using $10 \mathrm{~g} \mathrm{~L}^{-1}$ of the kelp solution with water. Culture was conducted in the bioreactor containing the $1 \mathrm{~L}$ buffered medium with $10 \mathrm{~g} \mathrm{~L}^{-1}$ of alginate and mannitol (1:2) as initial carbon sources. Especially, phosphate buffer concentration was adjusted to $20 \mathrm{mM}$ and $5 \mathrm{~g} \mathrm{~L}^{-1}$ of yeast extract were additionally provided. Sterile air at $2 \mathrm{~L} \mathrm{~min}^{-1}$ was provided during initial $6 \mathrm{~h}$. Then, the cells were cultured anaerobically and $20 \mathrm{~g} \mathrm{~L}^{-1}$ of the kelp powder was added with a $3 \mathrm{~h}$ interval. The culture was continuously stirred at $300 \mathrm{rpm}$.

Metabolite quantification. Sugars and metabolites (including ethanol and 2,3$\mathrm{BDO}$ ) were quantified using the UltiMate ${ }^{\mathrm{TM}} 3000$ analytical high-performance liquid chromatography system (Dionex, Sunnyvale, CA, USA) equipped with an Aminex HPX-87H column (Bio-Rad Laboratories). As a mobile phase, we used $5 \mathrm{mM}$ of sulfuric acid at a flow rate of $0.6 \mathrm{~mL} \mathrm{~min}^{-1}$ and $65^{\circ} \mathrm{C}$. The refractive index (RI) signal was monitored using a Shodex RI-101 detector (Shodex, Klokkerfaldet, Denmark).

Alginate was quantified using a modified version of a previously described method $^{40}$. Briefly, $200 \mu \mathrm{L}$ of the sample was mixed with $1 \mathrm{~mL}$ of $0.025 \mathrm{M}$ sodium tetraborate $10 \mathrm{H}_{2} \mathrm{O}$ in sulfuric acid. After cooling the mixture in an icebox, $40 \mu \mathrm{L}$ of $0.125 \%$ carbazole in absolute ethanol was added and the mixture gently was shaken. The absorbance at $530 \mathrm{~nm}$ was measured using the Hidex Sense microplate reader. The samples were appropriately diluted to fall within a $0-2 \mathrm{~g} \mathrm{~L}^{-1}$ range and a standard curve was prepared with using various concentrations of alginate solution $\left(0,0.05,0.2,0.5,1\right.$, and $\left.2 \mathrm{~g} \mathrm{~L}^{-1}\right)$.

To quantify lycopene production, lycopene from cell pellets was extracted by adding pure acetone. The absorbance of the samples was then measured at $475 \mathrm{~nm}^{41}$.

Pathogenicity analysis. Potential pathogenicity of Vibrio. sp dhg was evaluated by searching known Vibrio toxins (Ace, CT, MARTX, TDH, TRH, VCC, and Zot) using the Basic Local Alignment Search Tool (BLAST, https://blast.ncbi.nlm.nih. gov/Blast.cgi?PROGRAM=tblastn $)^{10,42,43}$. Amino acid sequence of the toxins and genome sequence of Vibrio sp. dhg were used as inputs.

Reporting summary. Further information on research design is available in the Nature Research Reporting Summary linked to this article.

\section{Data availability}

Data supporting the findings of this work are available within the paper and its Supplementary Information files. A reporting summary for this Article is available as a Supplementary Information file. The genome sequence of Vibrio sp. dhg has been uploaded to Genbank under accession number CP028943, СР028944, and CP028945. The transcriptomic data was deposited in Gene Expression Omnibus under accession number GSE119357. A reporting summary for this article is available as a Supplementary Information file. The source data underlying Figs. $1 \mathrm{~b}-\mathrm{d}, 2 \mathrm{a}-\mathrm{c}$ are provided as a Source Data file. The other datasets generated and analyzed during the current study are available from the corresponding authors upon request.

Received: 21 October 2018 Accepted: 8 May 2019

Published online: 06 June 2019

\section{References}

1. Biddy, M. J., Scarlata, C. \& Kinchin, C. Chemicals from Biomass: A Market Assessment of Bioproducts with Near-Term Potential. https://doi.org/10.2172/ 1244312 (National Renewable Energy Laboratory (NREL), 2016).
2. Kawai, S. \& Murata, K. Biofuel production based on carbohydrates from both brown and red macroalgae: recent developments inkey biotechnologies. Int. J. Mol. Sci. 17, 145 (2016).

3. Roesijadi, G., Jones, S. B., Snowden-Swan, L. J. \& Zhu, Y. Macroalgae as a Biomass Feedstock: A Preliminary Analysis. https://doi.org/10.2172/1006310 (Pacific Northwest National Laboratory, 2010).

4. Holdt, S. L. \& Kraan, S. Bioactive compounds in seaweed: functional food applications and legislation. J. Appl. Phycol. 23, 543-597 (2011).

5. Wei, N., Quarterman, J. \& Jin, Y.-S. Marine macroalgae: an untapped resource for producing fuels and chemicals. Trends Biotechnol. 31, 70-77 (2013).

6. Wargacki, A. J. et al. An engineered microbial platform for direct biofuel production from brown macroalgae. Science 335, 308-313 (2012).

7. Hehemann, J.-H. et al. Adaptive radiation by waves of gene transfer leads to fine-scale resource partitioning in marine microbes. Nat. Commun. 7, 12860 (2016).

8. Santos, C. N. S., Regitsky, D. D. \& Yoshikuni, Y. Implementation of stable and complex biological systems through recombinase-assisted genome engineering. Nat. Commun. 4, 2503 (2013).

9. Hoffart, E. et al. High substrate uptake rates empower Vibrio natriegens as production host for industrial biotechnology. Appl. Environ. Microbiol. 83, e01614-e01617 (2017).

10. Weinstock, M. T., Hesek, E. D., Wilson, C. M. \& Gibson, D. G. Vibrio natriegens as a fast-growing host for molecular biology. Nat. Methods 13, 849-851 (2016).

11. Petersen, T. N., Brunak, S., von Heijne, G. \& Nielsen, H. SignalP 4.0: discriminating signal peptides from transmembrane regions. Nat. Methods $\mathbf{8}$, 785-786 (2011)

12. Lim, H. G., Lim, J. H. \& Jung, G. Y. Modular design of metabolic network for robust production of $n$-butanol from galactose-glucose mixtures. Biotechnol. Biofuels 8, 137 (2015).

13. Contador, C. A. et al. Analyzing redox balance in a synthetic yeast platform to improve utilization of brown macroalgae as feedstock. Metab. Eng. Commun. 2, 76-84 (2015).

14. Registry of standard biological parts, Anderson promoter collection. http:// parts.igem.org/Promoters/Catalog/Anderson

15. Seo, S. W. et al. Predictive design of mRNA translation initiation region to control prokaryotic translation efficiency. Metab. Eng. 15, 67-74 (2013).

16. Seo, S. W. et al. Predictive combinatorial design of mRNA translation initiation regions for systematic optimization of gene expression levels. Sci. Rep. 4, 4515 (2014).

17. Lee, H. H., Ostrov, N., Gold, M. A. \& Church, G. M. Recombineering in Vibrio natriegens. Preprint at https://doi.org/10.1101/130088 (2017).

18. Chen, W., Ho, J. W., Huang, J. \& Watt, R. M. Functional characterization of an alkaline exonuclease and single strand annealing protein from the SXT genetic element of Vibrio cholerae. BMC Mol. Biol. 12, 16 (2011).

19. Datsenko, K. A. \& Wanner, B. L. One-step inactivation of chromosomal genes in Escherichia coli K-12 using PCR products. Proc. Natl Acad. Sci. USA 97, 6640-6645 (2000).

20. Schiener, P., Black, K. D., Stanley, M. S. \& Green, D. H. The seasonal variation in the chemical composition of the kelp species Laminaria digitata, Laminaria hyperborea, Saccharina latissima and Alaria esculenta. J. Appl. Phycol. 27, 363-373 (2015).

21. Mazumdar, S., Lee, J. \& Oh, M.-K. Microbial production of 2,3 butanediol from seaweed hydrolysate using metabolically engineered Escherichia coli. Bioresour. Technol. 136, 329-336 (2013).

22. Khan, N., Afaq, F. \& Mukhtar, H. Cancer chemoprevention through dietary antioxidants: progress and promise. Antioxid. Redox Signal. 10, 475-510 (2008).

23. Jung, J. et al. Precise precursor rebalancing for isoprenoids production by fine control of gapA expression in Escherichia coli. Metab. Eng. 38, 401-408 (2016).

24. Rost, B. Twilight zone of protein sequence alignments. Protein Eng. Des. Sel. 12, 85-94 (1999).

25. Di Pierro, M. et al. Zonula occludens toxin structure-function analysis. Identification of the fragment biologically active on tight junctions and of the zonulin receptor binding domain. J. Biol. Chem. 276, 19160-19165 (2001).

26. Liu, J., Li, H., Zhao, G., Caiyin, Q. \& Qiao, J. Redox cofactor engineering in industrial microorganisms: strategies, recent applications and future directions. J. Ind. Microbiol. Biotechnol. 45, 313-327 (2018).

27. Lim, J. H., Seo, S. W., Kim, S. Y. \& Jung, G. Y. Model-driven rebalancing of the intracellular redox state for optimization of a heterologous $n$-butanol pathway in Escherichia coli. Metab. Eng. 20, 56-62 (2013).

28. Lim, H. G., Jang, S., Jang, S., Seo, S. W. \& Jung, G. Y. Design and optimization of genetically encoded biosensors for high-throughput screening of chemicals. Curr. Opin. Biotechnol. 54, 18-25 (2018).

29. Kim, S. G. et al. Molecular parts and genetic circuits for metabolic engineering of microorganisms. FEMS Microbiol. Lett. 365, fny187 (2018). 
30. Mohamed, E. T. et al. Generation of a platform strain for ionic liquid tolerance using adaptive laboratory evolution. Microb. Cell Fact. 16, 204 (2017).

31. LaCroix, R. A., Palsson, B. O. \& Feist, A. M. A mode 1 for designing adaptive laboratory evolution experiments. Appl. Environ. Microbiol. 83, e03115-e03116 (2017).

32. Campbell, K., Xia, J. \& Nielsen, J. The impact of systems biology on bioprocessing. Trends Biotechnol. 35, 1156-1168 (2017).

33. Song, Y. et al. Determination of the genome and primary transcriptome of syngas fermenting Eubacterium limosum ATCC 8486. Sci. Rep. 7, 13694 (2017).

34. Lee, O. K., Hur, D. H., Nguyen, D. T. N. \& Lee, E. Y. Metabolic engineering of methanotrophs and its application to production of chemicals and biofuels from methane. Biofuels, Bioprod. Bioref. 10, 848-863 (2016).

35. Deatherage, D. E. \& Barrick, J. E. Identification of mutations in laboratoryevolved microbes from next-generation sequencing data using breseq. Methods Mol. Biol. 1151, 165-188 (2014).

36. Aziz, R. K. et al. The RAST Server: rapid annotations using subsystems technology. BMC Genom. 9, 75 (2008).

37. Andrews, S. Babraham Bioinformatics - FastQC, A quality control tool for high throughput sequence data. http://www.bioinformatics.babraham.ac.uk/ projects/fastqc/ (2010).

38. Langmead, B. \& Salzberg, S. L. Fast gapped-read alignment with Bowtie 2. Nat. Methods 9, 357-359 (2012)

39. Trapnell, C. et al. Transcript assembly and quantification by RNA-Seq reveals unannotated transcripts and isoform switching during cell differentiation. Nat. Biotechnol. 28, 511-515 (2010).

40. Bitter, T. \& Muir, H. M. A modified uronic acid carbazole reaction. Anal. Biochem. 4, 330-334 (1962).

41. Kang, C. W. et al. Synthetic auxotrophs for stable and tunable maintenance of plasmid copy number. Metab. Eng. 48, 121-128 (2018).

42. Chen, L. et al. VFDB: a reference database for bacterial virulence factors Nucleic Acids Res. 33, D325-D328 (2005).

43. Chen, L., Zheng, D., Liu, B., Yang, J. \& Jin, Q. VFDB 2016: hierarchical and refined dataset for big data analysis-10 years on. Nucleic Acids Res. 44, D694-D697 (2016).

\section{Acknowledgements}

This research was supported by the C1 Gas Refinery Program (NRF2018M3D3A1A01055754), the Global Research Laboratory Program (NRF2016K1A1A2912829), and the Bio \& Medical Technology Development Program (NRF2018M3A9H3020459) through the National Research Foundation of Korea (NRF) funded by the Ministry of Science and ICT. The Basic Science Research Program (NRF2018R1A6A3A11045727) through the NRF funded by the Ministry of Education and Creative-Pioneering Researchers Program through Seoul National University (SNU) also supported this research. We would especially like to thank Dr. Hwapyeong Jeong and Ha Hyeon Jeon for technical support including SEM imaging.

\section{Author contributions}

H.G.L., D.H.K. and S.H.W. isolated, characterized, and engineered Vibrio sp. dhg. S.P. prepared samples for high-throughput sequencing and analyzed the omics-data with J.S Y. C.W.K. carried out biochemical production and metabolite quantification. B.H.K. and M.H.N. contributed to genome sequencing and annotation of the assembled genome. H.G.L., D.H.K., S.P., S.H.W., S.W.S. and G.Y.J. wrote the manuscript together with the other authors. S.W.S. and G.Y.J. supervised the overall project. All authors approved the final version of the manuscript.

\section{Additional information}

Supplementary Information accompanies this paper at https://doi.org/10.1038/s41467019-10371-1.

Competing interests: H.G.L., D.H.K., S.W.S., and G.Y.J. are inventors on Korean patent application 10-2018-0054287 and PCT patent application PCT/KR2018/005437, filed by Pohang University of Science and Technology Research and Business Development Foundation and Seoul National University Research and Development Foundation. These patents are based on this work. All other authors declare no competing interests.

Reprints and permission information is available online at http://npg.nature.com/ reprintsandpermissions/

Journal peer review information: Nature Communications thanks Bastian Blombach, Shigeyuki Kawai and the other, anonymous, reviewer(s) for their contribution to the peer review of this work. Peer reviewer reports are available.

Publisher's note: Springer Nature remains neutral with regard to jurisdictional claims in published maps and institutional affiliations.

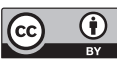

Open Access This article is licensed under a Creative Commons Attribution 4.0 International License, which permits use, sharing, adaptation, distribution and reproduction in any medium or format, as long as you give appropriate credit to the original author(s) and the source, provide a link to the Creative Commons license, and indicate if changes were made. The images or other third party material in this article are included in the article's Creative Commons license, unless indicated otherwise in a credit line to the material. If material is not included in the article's Creative Commons license and your intended use is not permitted by statutory regulation or exceeds the permitted use, you will need to obtain permission directly from the copyright holder. To view a copy of this license, visit http://creativecommons.org/ licenses/by/4.0/

(C) The Author(s) 2019 